\title{
Properties and Application Prospect of Boron Nitride Nanotubes
}

\author{
Youjun Wang, Hao Chen \& Yanjiao Li \\ Xi'an Research Institute of High Technology, Xi 'an 710025, China
}

ABSTRACT: Boron nitride nanotubes (BNNTs) is a new type of nano materials, with a variety of excellent physical and chemical properties. Based on the current research results, this paper makes a comprehensive summary of the relevant properties of BNNTs, and expounds its application field and development status.

KEYWORD: boron nitride nanotubes; application; property

\section{INTRODUCTION}

Since the discovery of carbon nanotubes (CNTs) by Lijima in 1991, it has attracted great interest of physicists, chemists and materials scientists. Boron nitride (BN) nanotubes and CNTs are similar, B atoms and $\mathrm{N}$ atoms in the form of a hybrid plane are similar to the structure of graphite, and boron nitride nanotubes (BNNTs) has some characteristics which CNTs does not have. After more than 20 years of research, people have in-depth study for the preparation methods and properties of the BNNTs, and Graspe the preparation of BNNTs method, at present, the mainly preparation method of BNNTs which have been reported include plasma arc discharge, laser ablation, chemical gas phase deposition method, ball-annealing method, template method and solvent thermal method.

BNNTs is the semiconductor with high bandwidth (about $5.5 \mathrm{ev}$ ) the conductive properties has no direct relationship between the diameter and helicity. BNNTs has higher temperature performance, better oxidation resistance, stronger resistance to chemical corrosion resistance, better thermal properties and better toughness than CNTs . These excellent properties make BNNTs have potential applications in many fields, such as semiconductor nano materials under high temperature, heat conduction of nano composite materials and insulation materials under high temperature.

\section{THE PROPERTIES OF BNNTS}

The structure of boron nitride nanotubes (BNNTs) is similar to carbon nanotubes (CNTs), B and N atoms placed staggered substitute $\mathrm{C}$ atoms, and the dis- tance of atomic almost haven't changed. Because of similar structure, BNNTs has some properties similar to CNTs, but because of the difference between BNNTs and CNTs in the electronic structure, BNNTs also showed some unique properties which not available in CNTs.

\subsection{Mechanical Property.}

Theoretical studies have shown that, BNNTs and CNTs are similar, also has a very high Young's modulus. However, compared to the CNTs (1.22$1.25 \mathrm{TPa}$ BNNTs, the young's modulus), the young's modulus of BNNTs is smaller (0.837$0.912 \mathrm{TPa}$ ).Chopra and Zettl tested the young's modulus of single multi walled BNNTs which was prepared by arc discharge, its value is as high as $1.22 \mathrm{TPa}$, close to single wall CNTs Young's modulus 1.26TPa (Young's modulus of Kevlar-49 fibers and SiC fibers were 112gpa and 200GPA), which is the highest in known insulating fiber. The results of Dumitrica and Yakobson showed that the activation energy of defects in BNNTs was lower than that of CNTs, but the formation energy was higher (T. Dumitrica et al, 2005). Therefore, under the normal temperature, the strength of CNTs is higher, in the high temperature environment, BNNTs is more stable and has higher strength instead.

In addition to a higher tensile strength and Young's modulus, BNNTs also has a good elastic plastic deformation capacity. Golberg (D Golberg et al, 2007)'s Experimental results show that, fixed BNNTs in high resolution transmission electron microscope (TEM) between two gold thread, after dozens of times of serious deformation, bending angle of about 70 degrees, BNNTs tube appeared severe bending and deformation. However, when the load is 
released, BNNTs is restored to its original perfect shape.

\subsection{Thermal Conductivity.}

Theoretical research shows that, the heat transmission of BNNTs and CNTs are very similar, the theoretical thermal conductivity are similar to CNTs, which is $60 \mathrm{~W} / \mathrm{MK}$; in 2006, Chang (C.W. Chang et al, 2006) measuring diameter $30 \sim 40 \mathrm{~nm}$ BNNTs at room temperature. Its thermal conductivity rate are $350 \mathrm{~W} / \mathrm{MK}$, also found that the thermal conductivity rate size of the BNNTs is dependent on the B isotopic disordered state, $\mathrm{K}$ value can be increased by $50 \%$ at room temperature, which may be the biggest in known material. Recently, Zettl (C.W. Chang et al, 2006) research group reported that the surface of the BNNTs can loading a large number of nonuniform heavy metal molecules, such as $\mathrm{C} 9 \mathrm{H} 16 \mathrm{Pt}$, such molecules with non axisymmetric heat conduction performance, and can produce a lot of heat fluxreduce in the direction which mass density discreased.

\subsection{Thermal Stability.}

The oxidation temperature of BNNTs which crystal intact and has less defects is 900 degrees Celsius, while at $500^{\circ} \mathrm{C}$, the CNTs which prepared by the CVD method has begun to rapidly oxidized. Recent studies show that the high purity multi walled BNNTs prepared at $1500 \mathrm{C}$ can maintain stability in air at about $1100^{\circ} \mathrm{C}$ (C. Zhi et al, 2005).

\subsection{Electrical Properties.}

Although the structure of BNNT and CNTs are very similar, their electrical properties are quite different. Theoretical calculations show that, regardless the diameter, the number of tube walls and the change of the number of chiral, BNNTs have a roughly uniform band gap width (about $5.5 \mathrm{eV}$ ). Theoretical calculations predict that the dielectric constant of BNNTs is 5.90 (H.P. Lan, et al, 2009), which usually shows good insulation properties.

The band structure of BNNTs can be changed by different methods. At present, the two methods of applied electric field and the addition of dopant have been verified by experiments, which can change the band gap of BNNTs. Cumings and Zettl experimental test the field emission properties of a single multi walled BNNTs, found that BNNTs showed obvious field emission current under lower voltage . It is also found that the electrical transport properties of BNNT can be improved significantly by adding $\mathrm{F}$ atoms and $\mathrm{C}$ atoms. For example, Tang $(\mathrm{C}$ Tang et al, 2005)"s study found that when the BNNT doped $\mathrm{F}$ atoms, the resistivity reduced from $300 \Omega \cdot \mathrm{cm}$ to $0.2 \sim 0.6 \Omega \cdot \mathrm{cm}$. Golberg studied the transport proper- ties of BNNT doped $\mathrm{C}$ atoms, and found that compared with the insulating property of BNNT and metallic property of CNT, the BNNT doped C atoms showed good semiconductor properties. In addition, the electron field emission, the field effect tube and the electronic rectification effect (L. Liao et al, 2007) are also observed in the BNNT doped C atoms. Bai (X Bai et al, 2007) first found the piezoelectric phenomenon of multi wall BNNTs, and verified by experiments.

\subsection{Optical Properties.}

Compared with other characteristics, a large number of theoretical and experimental research work on the luminescence and optical absorption properties of BNNTs were explored. A recent study shows that the optical absorption behavior of BNNTs is closely related to the exciton effect in the nanotube. However, most theoretical studies on the optical properties of BNNTs are based on the calculation of the band structure (G. Y. Guo et al, 2005). Studies show that the luminescence and absorption spectra of BNNT depend on their chiral and external transverse electric field.

Lauret Study on the optical absorption properties of the single wall BNNT which was prepared by laser sintering (J. S. Lauret et al, 2005). The optical transitions was observed in $4.45 \mathrm{eV}$ and $5.5 \mathrm{eV}$. The study of the photoluminescence and the characteristics of the cathode luminescence of BNNT shows, BNNTs is a material with strong violet and ultraviolet emission. Different samples, the study found that the emission peak is also slightly different. The reported at a wavelength of $230 \mathrm{~nm}, 279 \mathrm{~nm}, 338 \mathrm{~nm}$ and $460 \mathrm{~nm}$ indicated that the emission peak of (C. Tang et al, 2007). Chen found that the BNNTs doped $\mathrm{Eu}$ and $\mathrm{Yb}$ has strong luminescence characteristics which was prepared by ball milling and annealing (Hua Chen et al, 2007).

\subsection{Magnetic Properties.}

Theoretical study found (J. Wu et al, 2009), doped $\mathrm{C}, \mathrm{O}, \mathrm{V}, \mathrm{Cr}, \mathrm{Mn}, \mathrm{Ge}, \mathrm{F}, \mathrm{Be}$, and $\mathrm{H}$ can cause the BNNTs produce magnetic. Doping elements are different, the predicted saturation magnetic moment of each doped atom is also different from 1 to $3.98 \mathrm{~B}$. Most of the magnetic moments are derived from the doped elements rather than the $\mathrm{B}$ atoms and $\mathrm{N}$ atoms. For example, C doped BNNTs may lead to antiferromagnetic ordered semi conducting body, while in the CNTs doped B and N atoms are not magnetic. This further proves the difference between BNNTs and CNTs in electronic structure. As to why the doping can cause the BNNT to be magnetic, there is no experimental study. 


\subsection{Wetting Property.}

Yum and Yu studied the wetting properties of single BNNTs by analyzing the contact angle between BNNTs and different liquids (K Yum et al, 2006). These liquids include non polar bromide, polyethylene glycol, glycerol, and polar water. Their study found that the contact angle of BNNTs with these liquids was slightly larger than that of CNTs. By calculation, the surface tension of BNNTs is about $27 \mathrm{MN} / \mathrm{m}$, which is very close to the $27.8 \mathrm{CNTs}$ of $\mathrm{MN} / \mathrm{m}$.

Lee et al. (C H Lee et al, 2009) found that compared with BN film, BNNTs film showed a more obvious hydrophobicity, BN film contact angle of about 44-52 degrees, and the contact angle of BNNTs film reached 145-160 degrees, the hydrophobic effect is very obvious.

Potential application prospects of BNNTs

BNNTs excellent mechanical properties, high thermal conductivity, good insulation characteristics and high temperature stability is the preparation of composite ideal additives, especially in applications that require high temperature resistant and insulation, BNNTs has more advantages than CNTs.

BNNTs has excellent heat conduction performance, if the BNNTs thermal rectifier can be made, will have great potential for development in the nano scale heat exchanger, micro electronic processor, refrigerator and energy saving building, etc..

The inherent electrical properties of BNNTs have an important advantage in the application of electronic and mechanical devices, and it also plays an important role in the field of the potential applications of emission devices and molecular transport.

Characteristics of BNNTs: low density, chemical stability, good thermal stability and has a porous structure, the electrical properties are independent of the diameter, the chiral and the number of the pipe wall, and the hydrogen storage required less energy than the CNTs. Therefore, BNNTs can become a stable and lightweight hydrogen storage materials.

BNNTs is light, non-toxic, excellent chemical stability and biocompatibility which make it become a new type of nano materials in the field of biological medicine. BNNTs excellent piezoelectric properties, so that it can be applied to the preparation of induction and stimulation of cells of the nano biosensors.

BNNTs doped $\mathrm{C}$ has excellent field emission properties, compared with CNTs, BNNTs doped C has a more stable temperature and chemical stability. The C-doped BNNTs in flat panel display field emission devices, this have opened up a new world for the scanning tunneling microscope (STM) and atomic force scanning electron microscopy (AFM). The doped BNNTs also has photoluminescence properties, which makes the BNNTs expected to be used in optoelectronic devices.

\section{SUMMARY}

BNNTs is a kind of nanometer material with excellent comprehensive properties. It has broad application prospects in many fields. Although scientists has made some breakthroughs in the preparation of BNNTs in recent years, but in general, it has not been reported that a method for preparing BNNTs with higher purity and better crystallinity can be produced in large quantities (more than one time with a single output of more than $100 \mathrm{~g}$ )so far. The further research on the theory and practical application of BNNTs is restricted by the problem of large batch preparation, which is to be further studied and explored by scholars.

\section{REFERENCES}

C H Lee, J Drelich, Y K Yap. Superhydrophobicity of boron nitride nanotubes grown on silicon substrates[J]. Langmuir, 2009, 25(9): 4853-4860.

C. Tang, Y. Bando, C. Zhi, D. Golberg. Boron-oxygen luminescence centres in boron-nitrogen systems[J]. Chemical Communications, 2007, 7(44): 4599-4601.

C Tang, Y Bando, Y Huang, S Yue, C Gu, F F Xu, D Golberg. Fluorination and electrical conductivity of $\mathrm{BN}$ nanotubes[J]. Journal of the American Chemical Society, 2005, 127(18): 6552-6553.

C.W. Chang, A M Fennimore, A Afanasiev, D Okawa, T Ikuno, H Garcia, D Li, A Majumdar, A. Zettl, Isotope effect on the thermal conductivity of boron nitride nanotubes[J]. Phys. Rev. Lett., 2006, 97, 085901-085904

C.W. Chang, D. Okawa, A. Majumdar, A. Zettl. Solid-state thermal rectifier[J]. Science, 2006, 314, 1121-1124.

C. Zhi, Y. Bando, C. Tang, R. Xie, T. Sekiguchi and D. Golberg: Perfectly Dissolved Boron Nitride Nanotubes Due to Polymer Wrapping[J]. J. Am. Chem. Soc., 2005, 127(46): 15996-15997.

D Golberg, X Bai, M Mitome, C Tang, C Zhi, Y Bando. Structural peculiarities of in situ deformation of a multi-walled $\mathrm{BN}$ nanotube inside a high-resolution analytical transmission electron microscope[J]. Acta Mater., 2007, 55: 1293-1298.

G. Y. Guo, J. C. Lin. Systematic ab initio study of the optical properties of BN nanotubes[J]. Physical Review B, 2005, 71(16): 165402 (12pp).

H.P. Lan, L.H. Ye, S.A. Zhang, L.M. Peng, Transverse dielectric properties of boron nitride nanotubes by ab initio electric field calculations[J]. Appl. Phys. Lett. 2009, 94-18: 8311083110.

Hua Chen, Ying Chen, Chi Pui Li,,et al. Eu-doped boron nitride nanotubes as a nanometer-sized visible-light source [J]. Adv. Mater, 2007, 19: 1845-1848.

J. S. Lauret, R. Arenal, F. Ducastelle, A. Loiseau, M. Cau, B Attal-Tretout, E Rosencher, L Goux-Capes. Optical transitions in single-wall boron nitride nanotubes[J]. Physical Review Letters, 2005, 94(3): 037405 (4pp).

J. $\mathrm{Wu}, \mathrm{W}$. Zhang. Tuning the magnetic and transport properties of boron-nitride nanotubes via oxygen-doping[J]. Solid State Communications, 2009, 149(11-12):486-490.

K Yum, M F Yu. Measurement of wetting properties of individual boron nitride nanotubes with the wilhelmy method 
using a nanotube-based force sensor[J]. Nano Letters, 2006, 6(2): 329-333.

L. Liao, K. Liu, W. Wang, et al. Multiwall boron carbonitride/carbon nanotube junction and its rectification behavior[J]. J. Am. Chem. Soc., 2007, 129:9562-9563.

T. Dumitrica, B.I. Yakobson. Rare theory of yield in boron nitride nanotubes [J]. Phys. Rev. B, 2005, 72 (3): 035418

$\mathrm{X}$ Bai, D Golberg, Y Bando, C Zhi, C Tang, M Mitome, K Kurashima. Deformation-driven electrical transport of individual boron nitride nanotubes [J].Nano Letters, 2007, 7(3): 632-637. 